\title{
Intestinal Imaging: The Last Frontier
}

\author{
Rakesh Sinha ${ }^{1}$ \\ ${ }^{1}$ Department of Radiology, South Warwickshire NHS Foundation \\ Trust, Warwick CV34 5BW, United Kingdom
}

J Gastrointestinal Abdominal Radio ISGAR 2018;1:7-11

\section{Radiological Evaluation of the Small Bowel Conventional Radiology}

The conventional small bowel follow-through (SBFT) examination that was routinely practiced has been shown to be inadequate in the evaluation of the small intestine. A large multi-institutional prospective study showed that the SBFT had inadequate definition of $35 \%$ of the proximal ileum, $32 \%$ of the distal ileum, and $24 \%$ of the jejunum. ${ }^{1}$ It is quite clear that the traditional SBFT examination that involves a patient drinking barium followed by serial radiographs at regular intervals should be abandoned as more than a third of the small bowel is not adequately visualized. "The SBFT fails to apply the same principles of diagnostic care that are the rule in virtually all other areas of radiology"-for example, does a radiologist still perform a skull radiograph for suspected stroke?

Nevertheless, barium studies still hold a limited place in the radiologist's arsenal. If performed, they should be done together with fluoroscopic evaluation, where segments of the bowel are observed during peristalsis and manual compression. Some added modifications such as pneumocolon should be used to visualize the ileocaecal junction. Barium examinations are useful in the evaluation of intestinal infections as they can show irregularities of fold patterns and more importantly peristaltic abnormalities that are not readily appreciated on other imaging modalities.

The most accurate conventional radiologic method in the diagnosis of small-bowel obstruction, inflammatory bowel disease (IBD), gastrointestinal bleeding, and small intestinal neoplasms is the double-contrast enteroclysis (DCE). ${ }^{2}$ The advantages of DCE over SBFT are greater and more consistent distension of the bowel may highlight strictures or small lesions. Subtle mucosal disease may also be better appreciated on DCE examinations (-Fig. 1). The major disadvantages of enteroclysis are patient discomfort and higher radiation dosages. However, studies have shown that the technique of DCE especially with air-contrast technique provides similar diagnostic accuracy to capsule endoscopy. The technique of enteroclysis is also used in computed tomography (CT) and magnetic resonance imaging (MRI) examinations of the small intestine.

\begin{abstract}
Address for correspondence Rakesh Sinha, MBBS, MD, DNB, FRCR, FICR, Department of Radiology, South Warwickshire NHS Foundation Trust, Warwick CV34 5BW, United Kingdom (e-mail: rakslide@hotmail.com).
\end{abstract}

In recent times, newer modalities have emerged for investigation of intestinal disorders and diseases. Innovations in intestinal imaging have been driven by the demand for detailed clinical information, better diagnoses, and higher accuracy rates.

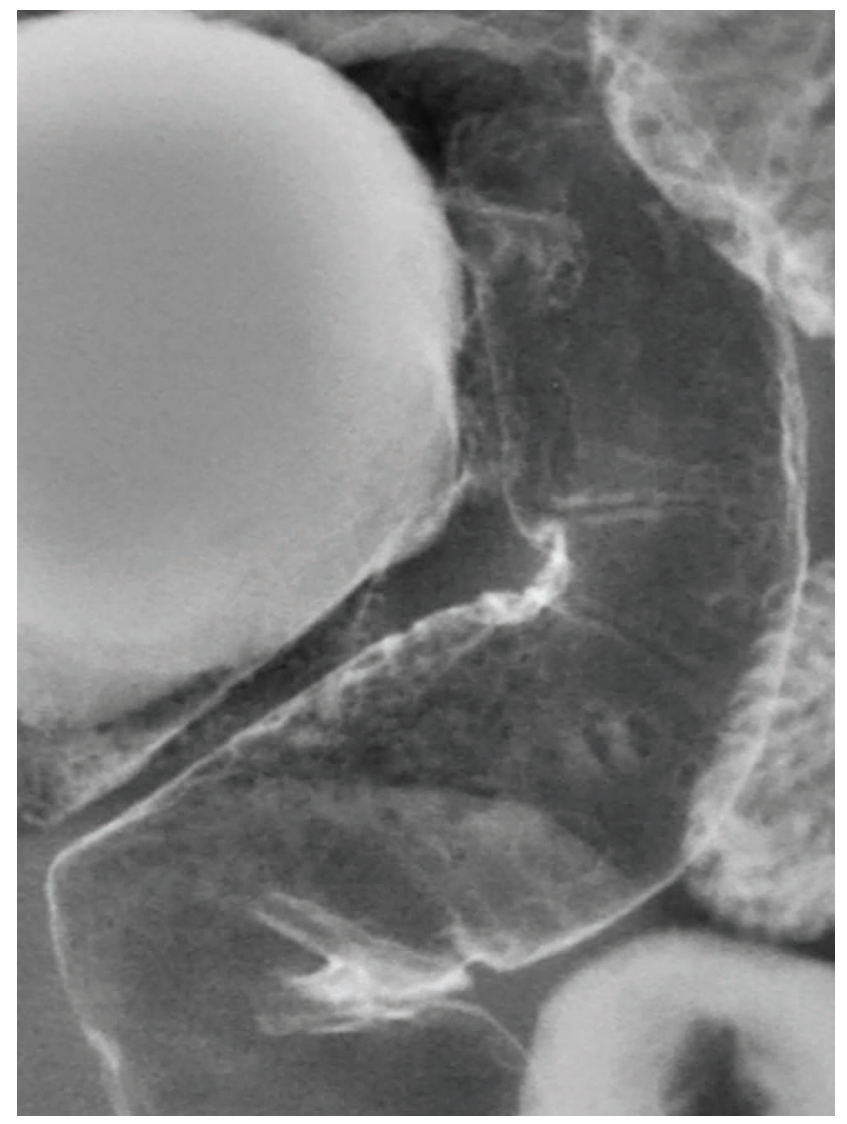

Fig. 1 Double-contrast enteroclysis (DCE) in a 36-year-old male patient with chronic diarrhea shows fine detail of the intestinal mucosa with aphthous ulcers, enlarged follicular nodules, and small, shallow ulcers in early Crohn's disease. These early changes may not be appreciated on routine computed tomography (CT) or magnetic resonance imaging (MRI) examinations.
License terms

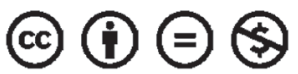




\section{CT and MR Imaging of the Small Bowel}

The small intestine can be imaged using the technique of enteroclysis combined with CT or MRI (CT enteroclysis and MR enteroclysis, respectively). Another technique for imaging the intestine is the enterography procedure (CT enterography [CTE] or MR enterography [MRE]).

A major advantage of CT- or MR-based examinations over traditional barium examinations are that they allow assessment of the bowel lumen, bowel wall, surrounding mesentery, and other abdominal organs. The major advantages of MR imaging over CT are its inherent tissue contrast resolution and absence of ionizing radiation. Real-time imaging for peristaltic activity and dynamic contrast studies are therefore possible with MRE without the risk of increased radiation burden. The major disadvantage of $\mathrm{CT}$ is its exposure to ionizing radiation which makes it unsuitable for pediatric population. (Please add here the advantages of CT over MRI). CT may be useful in the acute setting or the unwell patient who may not tolerate the longer MR examination. Better anatomical detail may also be achieved on CT due to its greater spatial resolution.

The fundamental principle behind obtaining diagnostic small intestinal images is good distension of the bowel lumen. Collapsed segments can even hide larger lesions and may appear falsely thickened or abnormally enhanced. Distension of the small intestine can be achieved via the enteroclysis of enterographic techniques. Although the enteroclysis technique can provide consistent and optimal distension, the procedure is technically challenging and discomfiting for patients. Furthermore, radiation is still involved during placement of the nasojejunal catheter. Small prospective studies have shown no difference in the diagnostic capabilities of MR enteroclysis and MRE studies. The enteroclysis technique is also more costly as it involves the use of the MR and fluoroscopy suites, time, staff, and nursing support.

Therefore, for practical purposes, the authors' choice for imaging the bowel is either CTE or MRE. The use of CT or MR enteroclysis is reserved for patients in whom the enterographic procedure has been suboptimal despite a high clinical index of suspicion. Enteroclysis may also be used in patients suspected of having partial strictures that may not be highlighted with enterography examinations. Invasive procedures like enteroclysis should ideally be used in selected problem-solving cases rather than a first-line test.

\section{Patient Preparation for Enterography}

Distention of the small intestine depends on patient compliance, volume of contrast ingested, and the timing of imaging. The intake of sufficient volumes of contrast medium combined with optimal timing of image acquisition is paramount for achieving good quality diagnostic images on CTE or MRE.

Currently, no consensus exists for volume of contrast required. In a study by Kuehle et al, it was observed that good distension of the bowel was achieved with $1,350 \mathrm{~mL}$ of contrast and that no additional benefit was achieved by increasing the contrast volume to $1,800 \mathrm{~mL}^{3}$ A study by Lohan et al reported that oral contrast reached the terminal ileum at a mean time of 55 minutes. ${ }^{4}$ The optimal time for imaging the entire small bowel has been reported to range between 50 and 60 minutes. ${ }^{4-6}$ Although there is no consensus on amount of contrast, experts agree that water should not be used as an enterographic agent. Several studies have reported water to be inadequate for distending the distal small bowel after oral intake ${ }^{5,7}$ Water may only be used during the enteroclysis procedure where the instillation of contrast is under the operator's direct control through the nasojejunal tube. Oral agents for CTE include methylcellulose, lactulose, mannitol, solutions containing locust-bean gum, or polyethylene glycol. ${ }^{7-9}$ Enteral agents work by retarding the resorption of water in the intestine and promoting luminal distention. A neutral density contrast should be preferred as high-density agents may mask mucosal abnormalities and mucosal enhancement ( - Fig. 2). Positive density contrast may be used in specific instances when filling defects or bands are suspected (such as polyps or nonsteroidal anti-inflammatory drug-related webs), as the dense contrast tends to highlight luminal abnormalities. However, for most practical purposes, neutral-density contrast is ideal for CTE examinations.

On MRE, contrast agents may be positive, that is, they produce increased signal intensity within the bowel lumen (gadolinium chelates), whereas negative agents cause a signal drop out (superparamagnetic particles). ${ }^{10}$ Biphasic
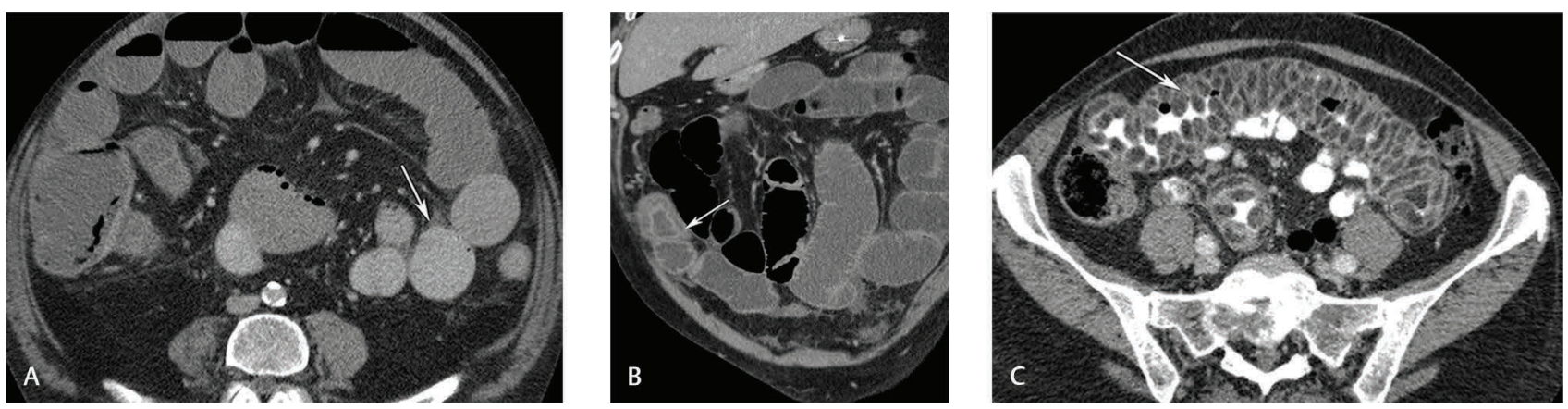

Fig. 2 Computed tomography enterography (CTE) examinations in a 44-year-old female patient. (A) Note positive enteral contrast obscures the mucosal enhancement (arrow). (B) CTE examination with neural-density contrast. The mucosal enhancement is clearly visible (arrow). (C) CTE examination with positive contrast in a 39-year-old patient with Whipple's disease. Note dense contrast highlights the thickened, nodular valvulae conniventes. 
agents (e.g., polyethylene glycol, mannitol solution) behave as positive or negative agents depending on the imaging sequence applied. At the authors' institution, a solution of $3 \%$ mannitol in 1,200 $\mathrm{mL}$ is used for CTE or MRE examinations.

It has been reported that a divided oral dose promotes a more uniform distension of the intestine. At our institution, the oral contrast material is divided in two aliquots of $600 \mathrm{~mL}$ each, and the patient drinks one aliquot every 25 to $30 \mathrm{~min}$ utes. An oral suspension of $10 \mathrm{mg}$ of metoclopramide is given with the first aliquot to promote gastric emptying. Continuous, steady ingestion of the oral contrast material over the allocated time promotes uniform and consistent filling of the proximal and distal small bowel ${ }^{11}$ ( - Fig. 3). The most important factor for promoting intestinal transit is a full stomach. ${ }^{12}$ Therefore, the addition of a second dose of oral contrast distends the stomach, promoting peristalsis and filling of the intestine. Just prior to acquiring images, patients are asked to drink another $200 \mathrm{~mL}$ of contrast material to outline the stomach and duodenum.

\section{CT and MR Imaging Sequences}

CTE imaging is performed with injection of iodinated contrast $(\sim 1-1.5 \mathrm{mg} / \mathrm{kg})$ at a rate of $4 \mathrm{~mL} / \mathrm{s}$ with a delay of 50 seconds. Images are acquired from the diaphragm to the symphysis pubis (-Fig. 2). An intravenous injection of $20 \mathrm{mg}$ of hyoscine-N- butylbromide (Buscopan) or $1 \mathrm{mg}$ of glucagon is administered as an antiperistaltic agent.

MRE is performed using ultra-fast sequences based on steady-state precession such as true-fast imaging with steady-state with free precession (true-FISP), balanced fast field echo, steady-state free precession, or fast imaging employing steady-state precession. These sequences need short, single breath-holds and are relatively insensitive to movement artifacts. These sequences provide high contrast between the bowel wall, lumen, and the mesentery. A disadvantage of these sequences is a black boundary artifact along the bowel wall which is often seen and can be minimized with fat suppression ( $\mathbf{- F i g s .} \mathbf{4}$ and $\mathbf{5}$ ).
MR images are also acquired using T2-weighted fast sequences such as half-Fourier acquisition single-shot turbo spin-echo (HASTE) or single shot fast spin echo. These sequences produce high contrast between the lumen and the bowel wall and are relatively insensitive to the black boundary artifact. Thin-section, high-resolution images using a combination of true FISP and HASTE sequences with fat suppression and small field of view may also be acquired parallel and perpendicular to the affected segments to maximize visualization of mucosal and mural abnormalities (-Figs. 5-7).

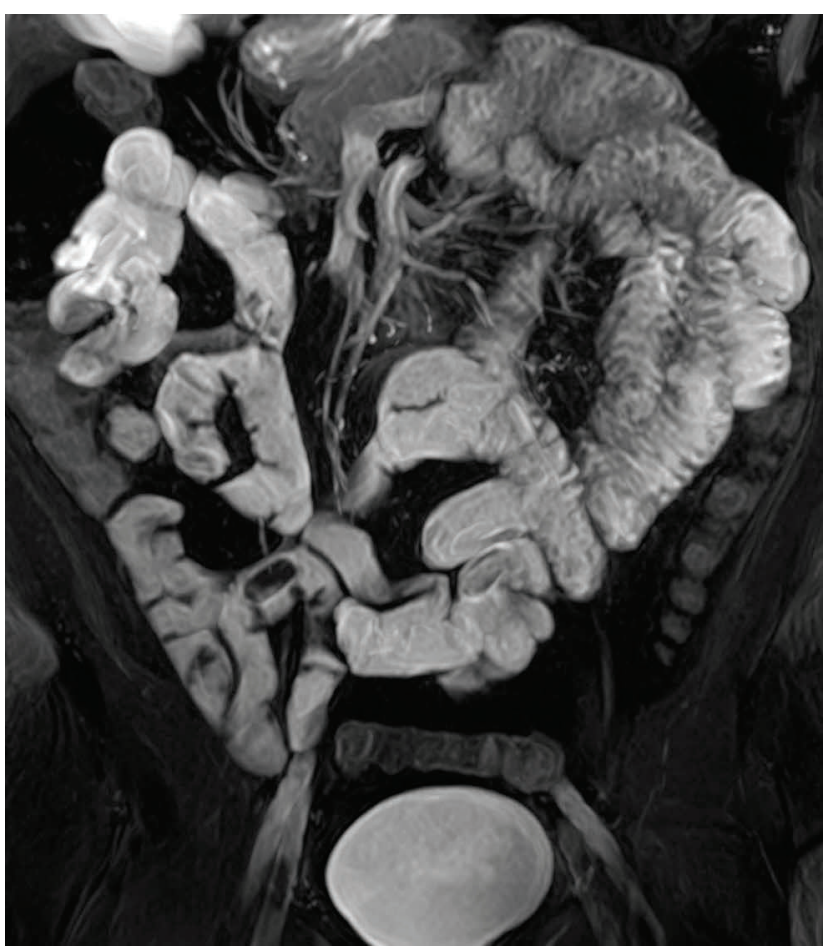

Fig. 3 True-fast imaging with steady-state free precession (FISP) coronal image from magnetic resonance enterography (MRE) examination in a 41-year-old male patient shows optimal distension of the small intestine up to the ileocaecal junction.
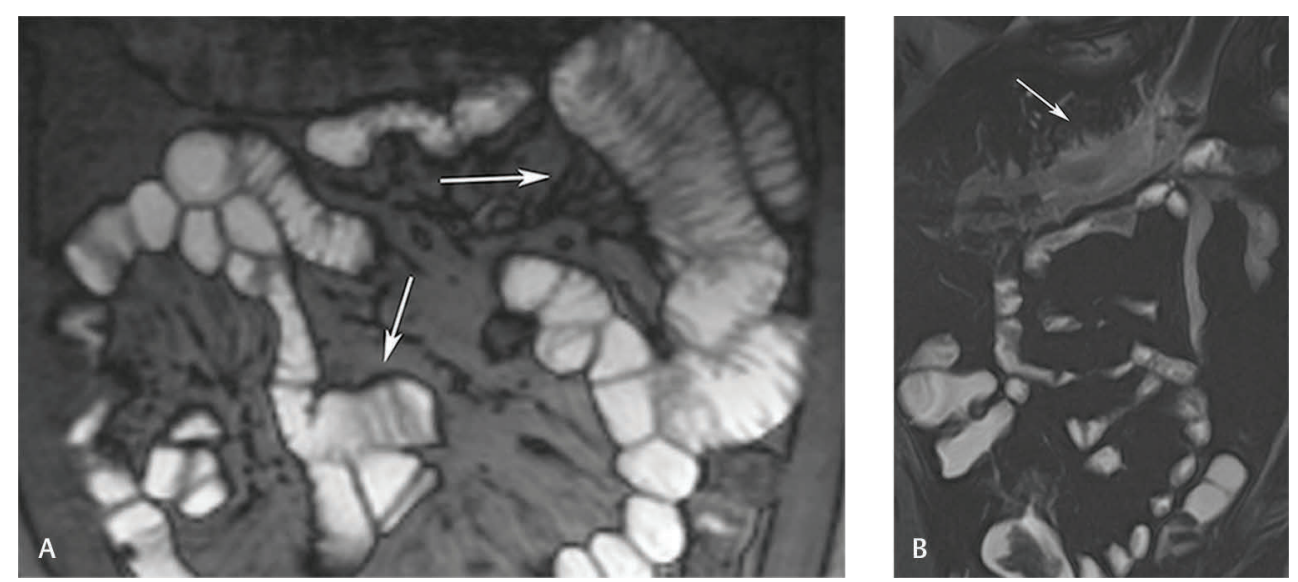

Fig. 4 Magnetic resonance enterography (MRE) examinations in a 36-year-old female patient. (A) Coronal true-fast imaging with steady-state free precession (FISP) image shows black-boundary artifact adjacent to the bowel wall (arrows). This artifact can obscure early mural changes. (B) Coronal true-FISP image in a 46-year-old male patient with fat suppression removes the artifact and early sinuses and fistulae from the inflamed transverse colon are easily appreciated (arrow). 


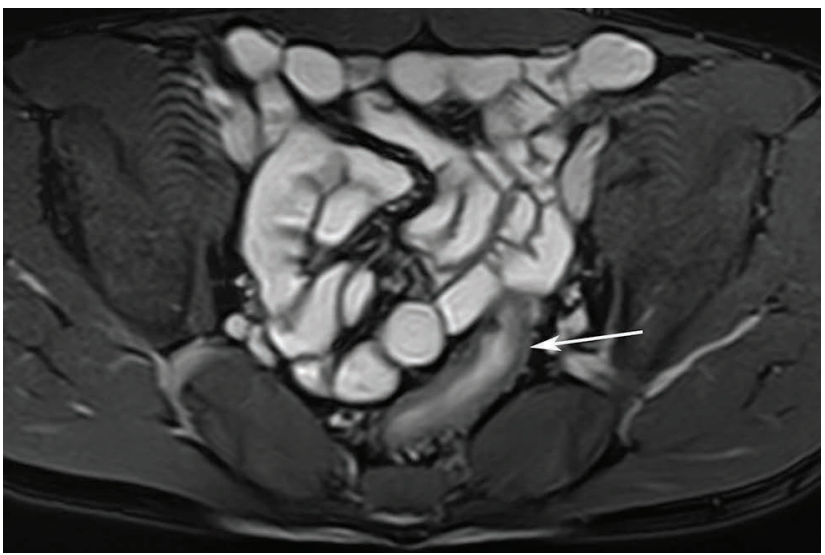

Fig. 5 True-fast imaging with steady-state free precession (FISP) axial image from magnetic resonance enterography (MRE) examination in a 21-year-old male patient shows marked mural thickening, ulcers, and inflammation of the bowel in Crohn's disease (arrow).

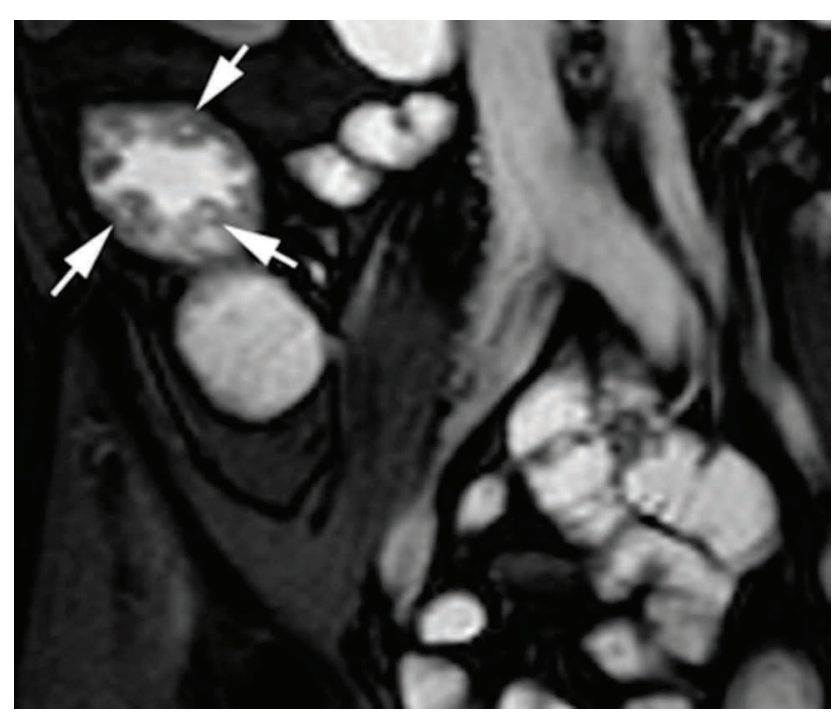

Fig. 6 High-resolution true-fast imaging with steady-state free precession (FISP) coronal image from magnetic resonance enterography (MRE) examination in a 31-year-old female patient shows marked nodular thickening of mucosal folds with target-type aphthous ulcers in Crohn's disease (arrows).

Postintravenous contrast images are acquired on T1-weighted sequences either in two or three dimensions. These sequences are commonly known as two-dimensional and three-dimensional (3D) fast low angle shot or volumetric sequences such as volumetric interpolated breath-hold examination. Fat saturation should be used to increase contrast resolution and also allows better assessment of bowel enhancement. ${ }^{5}$ Changes in bowel peristalsis can be evaluated on MRI fluoroscopy to demonstrate either an obstructive fibrotic or inflammatory stricture. ${ }^{13}$

\section{Diffusion-Weighted MR Imaging}

Diffusion-weighted (DW) MRI signal is derived from the motion of water molecules within cells or extracellular spaces. The use of high $b$ values ( $b=1,000$ second $/ \mathrm{mm}^{2}$ ) is recommended in DW MRI of the intestines to negate the high signal

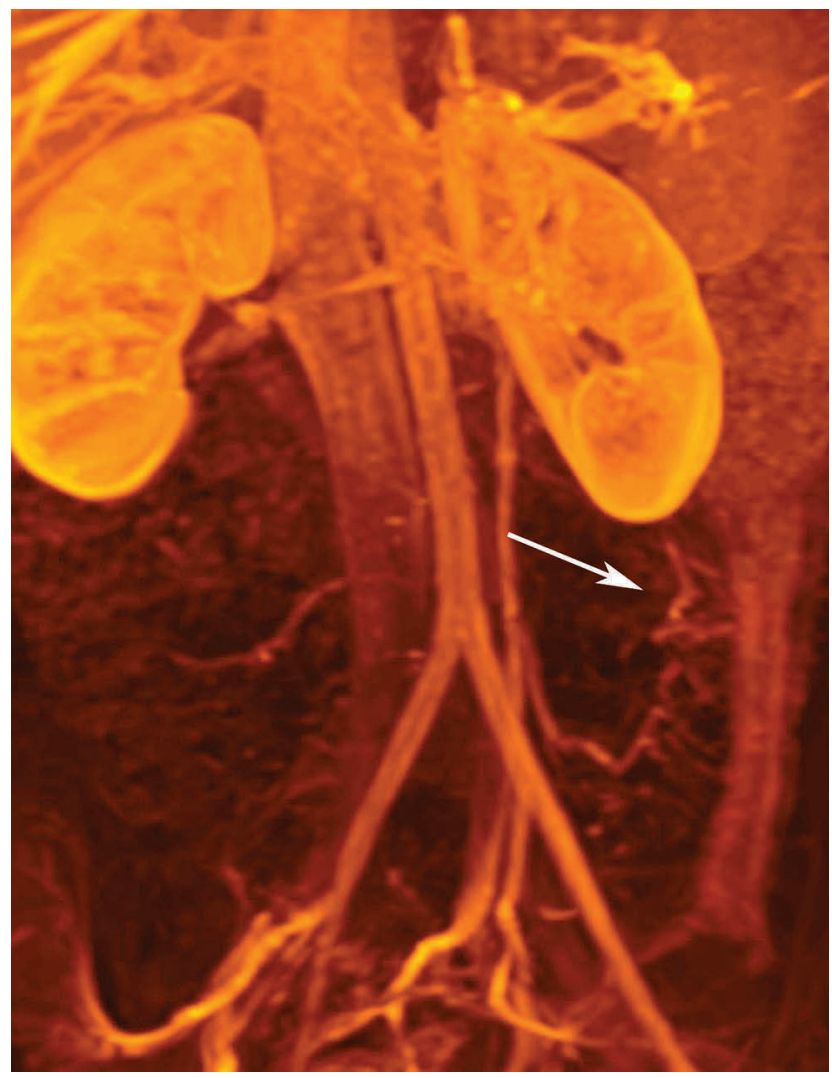

Fig. 7 Color-coded enhancement signal on magnetic resonance enterography (MRE) in a 22-year-old female patient shows hyperemia/perfusion in the descending colon with enlarged branches of the inferior mesenteric arcade (arrow). The lumen is narrowed due to an inflammatory stricture.

intensity of normal bowel mucosa and shine through effect from luminal contrast. ${ }^{14}$ Intestinal tumors or inflammation have restricted water diffusion and show up as areas retaining high signal intensity on high $b$ values.

The advantages of DW MR imaging are its noninvasive nature and no need for intravenous contrast injection. As DW MR imaging can be integrated with standard MRE imaging, this does not require any additional equipment. DW MR imaging uses the diffusion of water to produce images. It therefore provides functional, quantitative information at the cellular level that provides accurate assessment of intestinal inflammation, tumors, and also response to therapy ( - Fig. 8).

\section{Perfusion Imaging}

Perfusion CT is a technique that combines anatomy with assessment of vascular physiology. Analyses of tumor enhancement, tumor blood flow, blood volume, mean transit time, and permeability surface area product are evaluated. Perfusion CT demonstrates angiogenesis in tumors and is used in the assessment of colorectal tumors and their response to treatment. ${ }^{15}$

\section{Bowel Length Measurement}

Recent reports have described using MR imaging to measure intestinal length. ${ }^{16}$ Accurate measurements are important in 


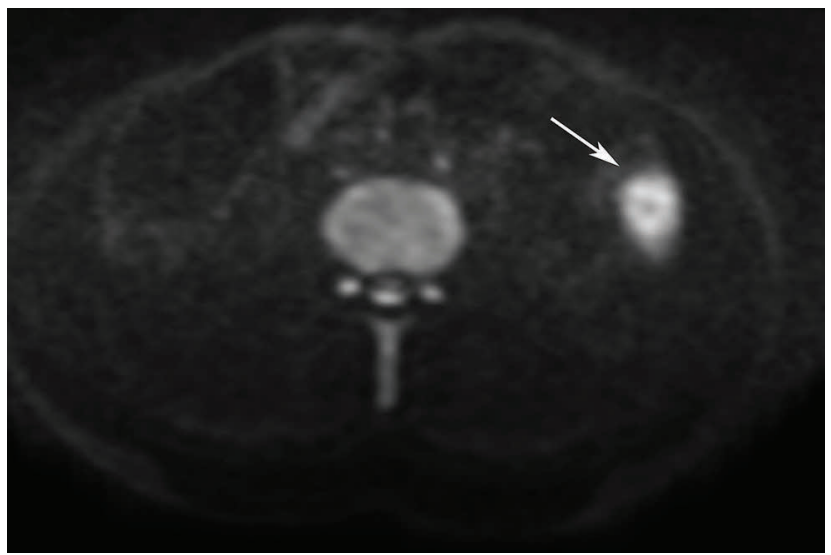

Fig. 8 Diffusion-weighted magnetic resonance imaging (MRI) in a 46-year-old male patient with colitis shows marked inflammation of the descending colon as area of transmural high signal (arrow).

surgical management for patients where repeated resections are required, such as in patients with Crohn's disease. Short intestinal length may lead to intestinal failure and extensive distal ileal resections lead to vitamin B12 deficiency and bile salt loss. There have been reports describing the use of software for small intestinal measurements. This is done using segmentation of MRE images using 3D directional gradient vector flow snakes with centerline extraction ( - Fig. 9). ${ }^{17}$

In conclusion, currently MRE has become the preferred technique for imaging of the small intestine. It is used in the diagnosis and follow-up of IBDs. ${ }^{18,19}$ MRE may also be used in pediatric population to avoid exposure to radiation. CTE may be used in patients who may not tolerate MRI examinations. Furthermore, CT imaging combined with enteroclysis is the most accurate diagnostic modality in the detection of partial strictures, adhesions, and tumors of the small bowel. New refinements such as perfusion imaging, bowel length measurement, and motility studies will further enhance the role of $C R$ and MR imaging of the small intestine in the future. ${ }^{20}$ In current practice, MR- or CT-based imaging should be the preferred choice for intestinal imaging rather than barium examinations.

\section{Conflict of Interest}

None.

\section{References}

1 Goldberg HI, Caruthers SB Jr, Nelson JA, Singleton JW. Radiographic findings of the National Cooperative Crohn's Disease Study. Gastroenterology 1979;77(4 Pt 2):925-937

2 Maglinte DD, Kelvin FM, O'Connor K, Lappas JC, Chernish SM. Current status of small bowel radiography. Abdom Imaging 1996;21(3):247-257

3 Kuehle CA, Ajaj W, Ladd SC, Massing S, Barkhausen J, Lauenstein TC. Hydro-MRI of the small bowel: effect of contrast volume, timing of contrast administration, and data acquisition on bowel distention. AJR Am J Roentgenol 2006;187(4):W375-85

4 Lohan D, Cronin C, Meehan C, Alhajeri AN, Roche C, Murphy J. MR small bowel enterography: optimization of imaging timing. Clin Radiol 2007;62(8):804-807

5 Sinha R, Verma R, Verma S, Rajesh A. MR enterography of Crohn disease: part 1, rationale, technique, and pitfalls. AJR Am J Roentgenol 2011;197(1):76-79

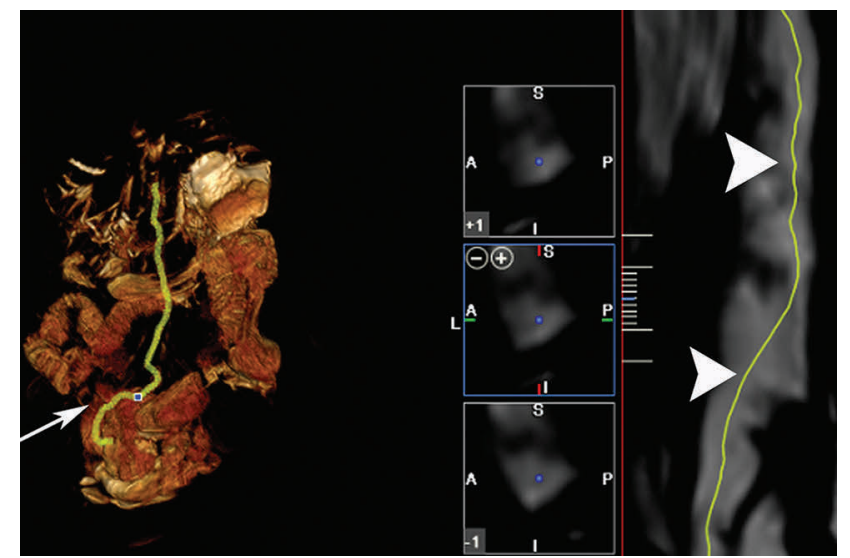

Fig. 9 Snapshot of a bowel length measurement with a vector snake (arrow). The length of bowel interrogated (arrowheads) is displayed prior to surgical planning.

6 Sinha R, Murphy P, Hawker P, Sanders S, Rajesh A, Verma R. Role of MRI in Crohn's disease. Clin Radiol 2009;64(4):341-352

7 Paulsen SR, Huprich JE, Hara AK. CT enterography: noninvasive evaluation of Crohn's disease and obscure gastrointestinal bleed. Radiol Clin North Am 2007;45(2):303-315

8 Macari M, Megibow AJ, Balthazar EJ. A pattern approach to the abnormal small bowel: observations at MDCT and CT enterography. AJR Am J Roentgenol 2007;188(5):1344-1355

9 Hara AK, Alam S, Heigh RI, Gurudu SR, Hentz JG, Leighton JA. Using CT enterography to monitor Crohn's disease activity: a preliminary study. AJR Am J Roentgenol 2008;190(6):1512-1516

10 Ajaj W, Debatin JF, Lauenstein T. Dark-lumen MR colonography. Abdom Imaging 2004;29(4):429-433

11 Sinha R, Rawat S. MRI enterography with divided dose oral preparation: Effect on bowel distension and diagnostic quality. Indian J Radiol Imaging 2013;23(1):86-91

12 Richards DG, Stevenson GW. Laxatives prior to small bowel follow-through: are they necessary for a rapid and good-quality examination? Gastrointest Radiol 1990;15(1):66-68

13 Sinha R. MR enterography. In: Konzarek R, Leighton JA, eds. Endoscopy of Small Bowel Disorders, New York. Springer; 2015

14 Sinha R, Rajiah P, Ramachandran I, Sanders S, Murphy PD. Diffusion-weighted MR imaging of the gastrointestinal tract: technique, indications, and imaging findings. Radiographics 2013;33(3):655-676, discussion 676-680

15 Figueiras RG, Goh V, Padhani AR, Naveira AB, Caamaño AG, Martin CV. The role of functional imaging in colorectal cancer. AJR Am J Roentgenol 2010;195(1):54-66

16 Sinha R, Trivedi D, Murphy PD, Fallis S. Small-intestinal length measurement on MR enterography: comparison with in vivo surgical measurement. AJR Am J Roentgenol 2014;203(3):W274-9

17 Summers RM, Swift JA, Dwyer AJ, Choi JR, Pickhardt PJ. Normalized distance along the colon centerline: a method for correlating polyp location on CT colonography and optical colonoscopy. AJR Am J Roentgenol 2009;193(5):1296-1304

18 Sinha R, Murphy P, Sanders S, et al. Diagnostic accuracy of high-resolution MR enterography in Crohn's disease: comparison with surgical and pathological specimen. Clin Radiol 2013;68(9):917-927

19 Sinha R, Verma R, Verma S, Rajesh A. MR enterography of Crohn disease: part 2, imaging and pathologic findings. AJR Am J Roentgenol 2011;197(1):80-85

20 Sinha R, Arumugam R. Crohn's Disease - Current Concepts. New York: Springer; 2015 